\title{
Superfluid-Helium Converter for Accumulation and Extraction of Ultracold Neutrons
}

\author{
O. Zimmer, ${ }^{1, *}$ K. Baumann, ${ }^{1}$ M. Fertl, ${ }^{1}$ B. Franke, ${ }^{1}$ S. Mironov,${ }^{1,2}$ C. Plonka, ${ }^{3}$ D. Rich, ${ }^{4,}$ P. Schmidt-Wellenburg,,${ }^{1,3}$ \\ H.-F. Wirth, ${ }^{1}$ and B. van den Brandt ${ }^{5}$ \\ ${ }^{1}$ Physik-Department E18, Technische Universität München, D-85748 Garching, Germany \\ ${ }^{2}$ Laboratory of Nuclear Problems, JINR, Dubna, Moscow Region 141980, Russia \\ ${ }^{3}$ Institut Laue-Langevin, BP 156, 38042 Grenoble, France \\ ${ }^{4}$ Forschungsreaktor München FRM II, Lichtenbergstrasse 1, 85747 Garching, Germany \\ ${ }^{5}$ Paul Scherrer Institut, CH-5232 Villigen PSI, Switzerland
}

(Received 27 May 2007; published 4 September 2007)

\begin{abstract}
We report the first successful extraction of accumulated ultracold neutrons (UCN) from a converter of superfluid helium, in which they were produced by downscattering neutrons of a cold beam from the Munich research reactor. Windowless UCN extraction is performed in vertical direction through a mechanical cold valve. This prototype of a versatile UCN source is comprised of a novel cryostat designed to keep the source portable and to allow for rapid cooldown. We measured time constants for UCN storage and extraction into a detector at room temperature, with the converter held at various temperatures between 0.7 and $1.3 \mathrm{~K}$. The UCN production rate inferred from the count rate of extracted $\mathrm{UCN}$ is close to the theoretical expectation.
\end{abstract}

DOI: 10.1103/PhysRevLett.99.104801

Introduction. - Ultracold neutrons (UCN) play an important role in fundamental investigations in particle physics and cosmology. Searches for the neutron electric dipole moment investigate $C P$ violation beyond the standard model of particle physics [1,2]. Accurate knowledge of the neutron lifetime is required for understanding big bang nucleosynthesis [3] as well as the semileptonic weak interaction within the first quark family (see, e.g., contributions to a recent workshop in Ref. [4]). Among other applications of UCN, the observation of quantum states of the neutron in the gravitational field of the earth has attracted recent interest [5]. The currently best source at the Institut Laue-Langevin in Grenoble [6] provides UCN with densities not exceeding a few 10 per $\mathrm{cm}^{3}$, which has motivated new source projects in various places around the world [7-13].

As pointed out long ago, superfluid ${ }^{4} \mathrm{He}$ can be used as a converter for UCN production in a superthermal cooling process of cold neutrons [14]. As a result of the crossing dispersion relations of superfluid ${ }^{4} \mathrm{He}$ and the free neutron, neutrons with wavelengths around $0.89 \mathrm{~nm}$, i.e., $1.0 \mathrm{meV}$ kinetic energy, can be scattered down to the ultracold energy range with emission of a single phonon. Multiphonon processes may also contribute, depending on the neutron spectrum incident on the converter $[15,16]$. Pure ${ }^{4} \mathrm{He}$ has no neutron absorption cross section, and at low temperature the density of excitations within the helium is so small that upscattering of UCN back to higher energy becomes unlikely. The UCN storage time $\tau$ may attain several $100 \mathrm{~s}$ if the converter vessel is made of a material with low UCN loss probability. Ideally, it is surrounded with a magnetic trap as used in the neutron lifetime experiment [17], where, for neutrons in the lowfield seeking spin state, $\tau$ may approach the neutron life-
PACS numbers: $29.25 . \mathrm{Dz}, 28.20 . \mathrm{Fc}, 61.12 . \mathrm{Ha}, 78.70 . \mathrm{Nx}$

time $\tau_{n}=885.7(8) \mathrm{s}$ [18]. Past experiments have already demonstrated UCN production rates in superfluid helium close to the theoretical expectation [12,17,19]. It was concluded that using an intense cold neutron beam available at a high flux source one might realize UCN densities up to several $10^{3}$ per $\mathrm{cm}^{3}$. Vertical windowless extraction of UCN from a superfluid-helium bath and their subsequent detection at room temperature was already demonstrated twice, first in an early experiment performed at the Institut Laue-Langevin in Grenoble [19], and recently by a Japanese group using a spallation neutron source [11].

The versatility of a superthermal helium UCN source would be strongly improved if one could accumulate the UCN prior to their extraction, in order to build up a high density. This was attempted 20 years ago [20], using a flap valve situated in the helium bath for horizontal UCN extraction. However, the scheme failed to be efficient, probably due to gaps and foils in the UCN transmission line, needed for thermal protection of the helium converter. Although the measured rate of upscattered neutrons was close to expectation, the rate of extracted UCN was a factor of 50 low. In turn, several groups decided to perform their experiments within the superfluid helium without extracting them.

Here we report the first successful extraction of UCN from superfluid helium after accumulation in the converter. Using a cold mechanical UCN valve situated above the helium bath, no gaps or windows are required. The small prototype involving a new type of cryostat enabled us to measure, with negligible background, the UCN production rate and to study the temperature-dependent storage properties of the converter. These first experiments are very promising for versatile applications on a larger scale. 
Apparatus. - The central pieces of the apparatus are the UCN converter vessel with a cold valve and connected tubing for UCN extraction (see Fig. 1). The present prototype has a rather small volume of about 2.41 . It is made from electropolished stainless steel tubes (from the milk industry) with total length $696 \mathrm{~mm}$ and inner diameter $66 \mathrm{~mm}$ and a neutron Fermi potential of 184(4) neV. This defines, after subtraction of the Fermi potential of the superfluid helium $\left(V_{F}=18.5 \mathrm{neV}\right)$, the maximum kinetic energy of storable neutrons. The incident cold neutron beam for UCN production passes through two $0.125 \mathrm{~mm}$ thick Ni foils $\left(V_{F}=252 \mathrm{neV}\right)$ which close off the vessel on both sides. The valve for UCN extraction is situated above the superfluid helium in a " $T$ " section of the tube. It can be manipulated from outside via a bellows-sealed capillary. With the valve open, UCN may exit through a $7 \mathrm{~cm}$ long vertical pipe with inner diameter $16 \mathrm{~mm}$. The subsequent extraction line consists of tapered transitions to diameter $50 \mathrm{~mm}$, followed by a $90^{\circ}$ bend, then a horizontal $30 \mathrm{~cm}$ long straight guide, a conical section expanding to diameter $66 \mathrm{~mm}$, then another $90^{\circ}$ bend, and finally a vertical $1 \mathrm{~m}$ long straight section down to a ${ }^{3} \mathrm{He}$ gas UCN detector. All UCN guides are made from electropolished stainless steel.

For filling and cooling the converter we developed a new cryostat. Primary cooling power is provided by a commercial two-stage Gifford McMahon cold head with a cooling power of $1.5 \mathrm{~W}$ at $4.2 \mathrm{~K}$. It cools the thermal radiation shields and liquefies helium from external gas supplies. Three separate systems are thermally connected in cascade to the cold head: (1) a continuous ${ }^{4} \mathrm{He}$ evaporation stage to reach a temperature below the $\lambda$ transition to superfluidity, (2) a closed ${ }^{3} \mathrm{He}$ system to reach $\lesssim 0.7 \mathrm{~K}$, and (3) a ${ }^{4} \mathrm{He}$ filling line for the UCN converter. The heat exchangers and condensers made from capillaries were already described in Ref. [21]. The ${ }^{4} \mathrm{He}$ for the UCN converter is supplied by a commercial gas cylinder and is $99.999 \%$ pure. To avoid capture of UCN by residual ${ }^{3} \mathrm{He}$, the liquefied helium is passed through a superleak held below the $\lambda$-transition

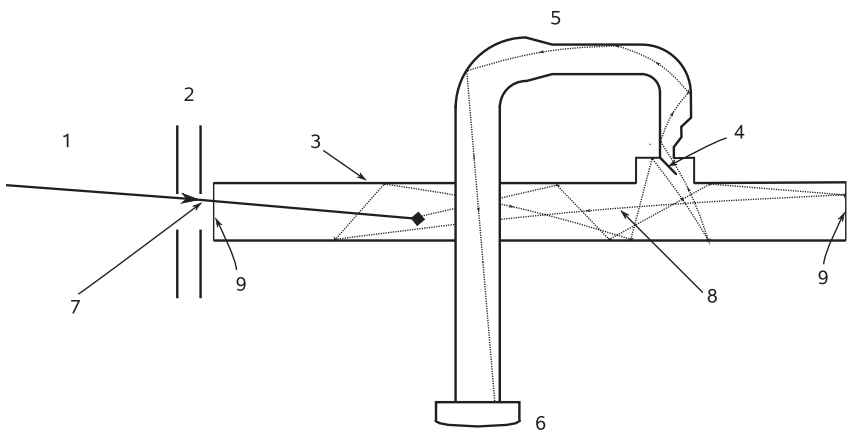

FIG. 1. Schematics of the apparatus for UCN production in superfluid ${ }^{4} \mathrm{He}$ : 1 cold neutron beam; 2 beam collimation; 3 stainless steel converter vessel; 4 flap valve for UCN extraction; $5 \mathrm{UCN}$ extraction guide; $6 \mathrm{UCN}$ detector; 7 cold neutron converted into a UCN; 8 trapped UCN, 9 Ni foils. temperature by the ${ }^{4} \mathrm{He}$ evaporation stage, which is supplied with liquefied helium through a needle valve. The superleak consists of a stainless steel tube with inner diameter $7 \mathrm{~mm}$, filled with a $17 \mathrm{~cm}$ long column of compressed $\mathrm{Al}_{2} \mathrm{O}_{3}$ powder with grain size $50 \mathrm{~nm}$. The pure ${ }^{4} \mathrm{He}$ then enters a heat exchanger connected to the ${ }^{3} \mathrm{He}$ evaporation stage. The interface is made from a cylindrical copper disk with holes increasing the total surface to $200 \mathrm{~cm}^{2}$ on each side. Cooled close to the temperature of the liquid ${ }^{3} \mathrm{He}$, the ${ }^{4} \mathrm{He}$ flows to the converter vessel through a $16 \mathrm{~cm}$ long tube with inner diameter $1 \mathrm{~cm}$. Exploiting the high heat conductivity of the superfluid, this results in a negligible temperature gradient. Using a roots blower with $500 \mathrm{~m}^{3} / \mathrm{h}$ nominal pumping speed backed by a $40 \mathrm{~m}^{3} / \mathrm{h}$ multiroots pump within the closed ${ }^{3} \mathrm{He}$ cycle, we were able to cool the filled converter down to $0.7 \mathrm{~K}$. The temperature was measured with a calibrated Cernox resistor attached to the converter volume. More details about the cryostat will be published elsewhere.

Experiments and results. - The apparatus was installed $1.7 \mathrm{~m}$ behind the exit of the cold neutron guide "NL1" at the Munich research reactor FRM II. The beam was collimated from diameter $60 \mathrm{~mm}$ down to diameter $33 \mathrm{~mm}$ at the entrance to our apparatus, thus defining a UCN production volume of $V_{p}=595 \mathrm{~cm}^{3}$. The neutron particle flux density determined there by gold foil activation was $1.5 \times 10^{9} \mathrm{~cm}^{-2} \mathrm{~s}^{-1}$ for a mean neutron wavelength of $0.53 \mathrm{~nm}$.

In "buildup mode" measurements we first irradiated the converter with cold neutrons with the UCN valve closed for an accumulation time $t_{0}$, after which the beam was shut under simultaneous opening of the valve for UCN detection. Figure 2 shows measured time histograms of UCN count rates at temperature $T=1.26 \mathrm{~K}$. Their integrals,

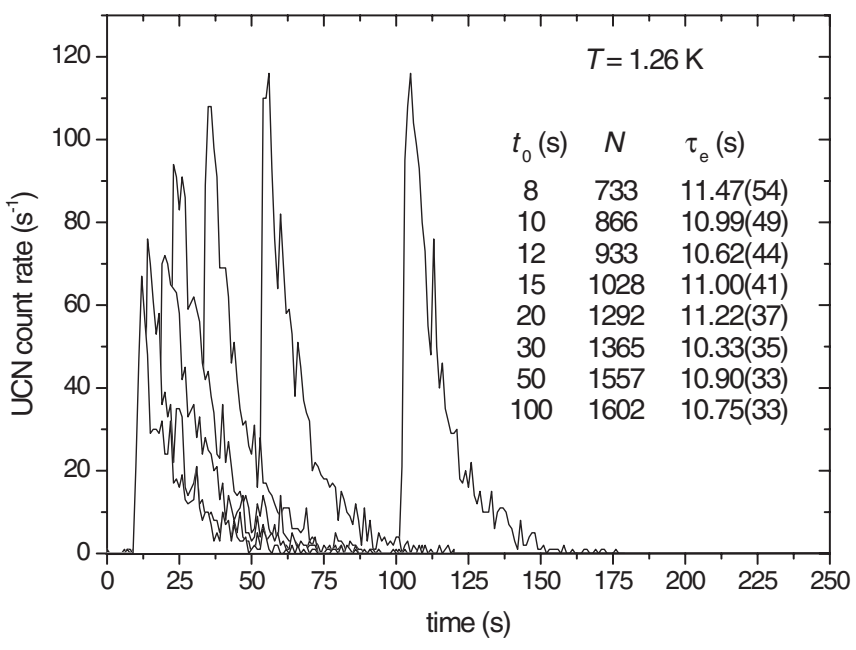

FIG. 2. Time histograms of UCN count rates, measured in buildup mode at $1.26 \mathrm{~K}$ for various $\mathrm{UCN}$ accumulation times $t_{0}$. Cold neutron irradiation starts at $t=0 . N$ denotes the integrated UCN counts for each of the histograms and $\tau_{e}$ the corresponding emptying time constant (see text). 
$N\left(t_{0}\right)$, follow nicely a simple exponential saturation function $\propto 1-\exp \left(-t_{0} / \tau\right)$. From the fit we obtained $\tau=$ $(13.28 \pm 0.65) \mathrm{s}$, which is consistent with an earlier measurement at this temperature using a different method [22]. The time constant $\tau$ for UCN buildup is identical to the storage time constant at closed UCN valve, which was also checked in a separate experiment. The rate $\tau^{-1}$ has a $T$-independent but UCN energy-dependent contribution $\tau_{0}^{-1}$ due to wall collisions, absorbing impurities, and UCN escape through small holes in the vessel, and a $T$-dependent contribution $\tau_{\text {up }}^{-1}$ due to UCN upscattering:

$$
\tau^{-1}=\tau_{0}^{-1}(E)+\tau_{\text {up }}^{-1}(T) .
$$

A second experimentally accessible quantity is the emptying time constant $\tau_{e}$ deduced from the exponential decrease in each of the histograms. It is related to $\tau$ and the time constant $\tau_{A}$ for UCN passage through the extraction hole with area $A$ and the consecutive guides by

$$
\tau_{e}^{-1}(T, E)=\tau^{-1}(T, E)+\tau_{A}^{-1}(E) .
$$

This follows from the proportionality of the detected rate to the UCN density in the vessel, which decreases due to storage losses and UCN extraction. At $T=1.26 \mathrm{~K}, \tau_{e}$ was found to be independent of $t_{0}$, with an average value $\tau_{e}=(10.82 \pm 0.14) \mathrm{s}$. This is consistent with a single time constant $\tau$ being sufficient to describe UCN buildup in the vessel. These observations tell us that, at this high temperature, $\tau^{-1}$ is dominated by the UCN energyindependent upscattering in the helium.

Results of measurements at $0.72 \mathrm{~K}$ show a much longer UCN buildup (see Fig. 3 and compare with Fig. 2). From a

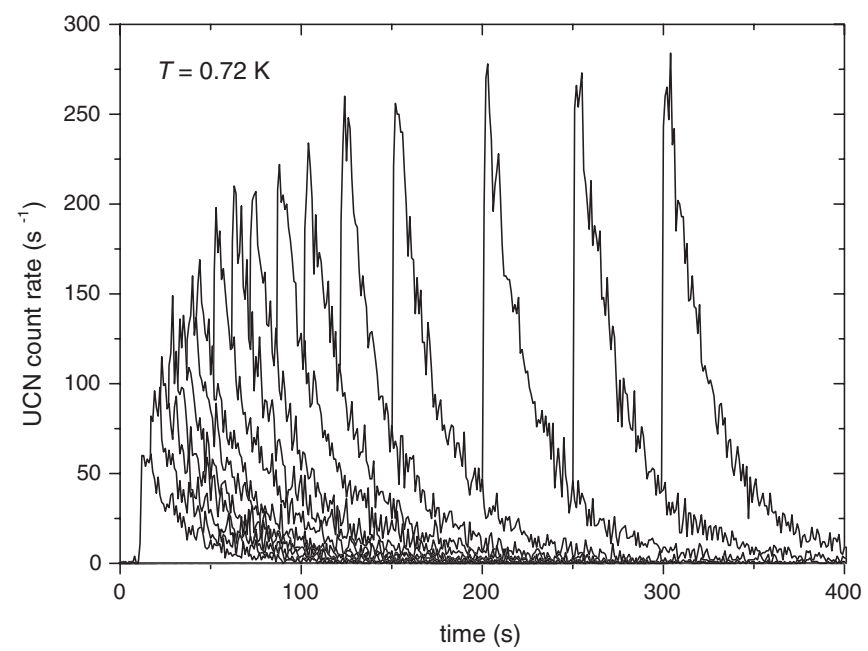

FIG. 3. Time histograms of UCN count rates, measured in buildup mode at $0.72 \mathrm{~K}$ for various accumulation times $t_{0}$. The corresponding emptying time constants $\tau_{e}$ are shown in Fig. 4. Note that the UCN detector was always recording events starting from $t=0$. The few counts for $0<t<7 \mathrm{~s}$ are due to all measurements in this time interval, which demonstrates the excellent background conditions. fit of the single- $\tau$ saturation function to the integrals one obtains $\tau=(55 \pm 2) \mathrm{s}$. Taking only the data for $t_{0} \geq$ $100 \mathrm{~s}$ results in $\tau=(69 \pm 1) \mathrm{s}$. This demonstrates that a single exponential fit to the whole data is no longer appropriate. An increase with accumulation time $t_{0}$ is also observed for $\tau_{e}$ (see Fig. 4, where results for other temperatures are also shown). The effects can be explained by UCN collisions with the walls of the converter vessel: losses are more significant for higher UCN energy (see, e.g., [23,24]), leading to a reduction of the mean UCN velocity. This results in smaller losses due to wall collisions and slower extraction after longer accumulation.

In "continuous mode" experiments we irradiated the converter with cold neutrons while the UCN valve was open. At $1.26 \mathrm{~K}$, the UCN rate was $\dot{N}_{c}=(100 \pm 5) \mathrm{s}^{-1}$. Defining $W=\varepsilon \tau_{A}^{-1} / \tau_{e}^{-1}=(0.185 \pm 0.041) \varepsilon$ as the probability for a created UCN to become detected, with the factor $\varepsilon$ accounting for imperfect detection efficiency and losses in the UCN guide, we may determine the UCN production rate density

$$
P=\frac{\dot{N}_{c}}{V_{p} W}=(0.91 \pm 0.21) / \varepsilon \mathrm{s}^{-1} \mathrm{~cm}^{-3} .
$$

Because of the negligible count rate with closed UCN valve no background correction of $\dot{N}_{c}$ was needed.

The production rate density expected from the 1-phonon process in a helium converter with $\mathrm{Be}$ wall coating $\left(V_{F}=252 \mathrm{neV}\right) \quad$ is $\quad P_{I}=(4.55 \pm 0.25) \times 10^{-9} d \phi /$ $\left.d \lambda\right|_{\lambda^{*}} \mathrm{~s}^{-1} \mathrm{~cm}^{-3}$, with the differential flux at $\lambda^{*}=$ $0.89 \mathrm{~nm}$ given in $\mathrm{cm}^{-2} \mathrm{~s}^{-1} \mathrm{~nm}^{-1}$ [12]. Using the results of prior time-of-flight (TOF) measurements of the beam at the exit of NL1 [25] and normalizing the spectrum with our

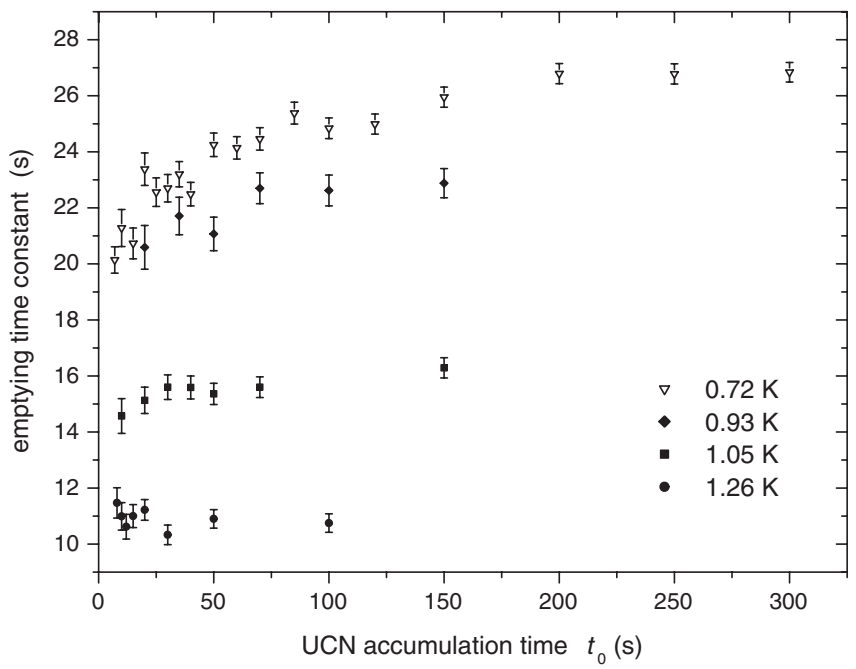

FIG. 4. Emptying time constants $\tau_{e}$ as a function of the UCN accumulation time $t_{0}$ in "storage mode" measurements for various temperatures. Note that in the measurements at $0.93 \mathrm{~K}$ the converter vessel was only partly filled, leading to an increased $\tau_{e}$ and larger counting statistical uncertainties. 
gold foil activation measurement of the integral flux, we obtain $d \phi /\left.d \lambda\right|_{\lambda^{*}}=5.0 \times 10^{8} \mathrm{~cm}^{-2} \mathrm{~s}^{-1} \mathrm{~nm}^{-1}$. Including a contribution of $30 \%$ to UCN production due to multiphonon processes in addition to $P_{I}$, as determined in Ref. [12] for a cold beam with similar spectrum, and dividing by 1.68 to account for the reduction of UCN phase space due to the lower Fermi potential of stainless steel, we might expect $P=1.76 \mathrm{~s}^{-1} \mathrm{~cm}^{-1}$. However, this value is definitely an overestimation, as the preceding analysis relies on earlier TOF measurements, which were performed with a filling of the reactor's cold source with 10.61 liquid deuterium. In the present measurements, it was operated with less than 91 , which leads to an intensity reduction notably of the long-wavelength part of the spectrum (this effect is described in Ref. [25]). In addition, divergence losses of $0.89 \mathrm{~nm}$ neutrons in the beam collimation are more severe than for shorter wavelengths. Both effects are not fully taken into account by normalization with our gold foil measurement. They are difficult to quantify without a dedicated TOF measurement planned for future experiments. With this uncertainty, our result for $P$ is indeed close to the expected value.

Conclusion. - In summary, we have for the first time successfully extracted ultracold neutrons accumulated in a converter of superfluid helium. Our setup provides excellent background conditions, which has enabled us to perform detailed investigations of storage and emptying time constants. These studies will be extended to lower temperatures and other storage materials in forthcoming experiments. With an upgraded apparatus versatile applications for experiments with UCN in vacuum are within reach. A specific application is the measurement of the neutron lifetime using a magnetic trap, for which one may also employ a different method of UCN extraction from the helium [26].

We would like to thank Christian Hesse for his help with the installation of equipment after midnight. This work is funded by BMBF under Contract No. 06MT250.

*oliver.zimmer@ph.tum.de
${ }^{\dagger}$ Current address: Oak Ridge National Laboratory, PO Box 2008, Oak Ridge, TN 37831, USA.

[1] C. A. Baker et al., Phys. Rev. Lett. 97, 131801 (2006).

[2] M. Pospelov and A. Ritz, Ann. Phys. (N.Y.) 318, 119 (2005).

[3] R.E. Lopez and M.S. Turner, Phys. Rev. D 59, 103502 (1999).

[4] Special issue on Precision Measurements with Slow Neutrons, edited by M. Arif et al. [J. Res. Natl. Inst. Stand. Technol. 110, 137 (2005)].

[5] V. V. Nesvizhevsky et al., Nature (London) 415, 297 (2002).

[6] A. Steyerl et al., Phys. Lett. A 116, 347 (1986).

[7] U. Trinks, F. J. Hartmann, S. Paul, and W. Schott, Nucl. Instrum. Methods Phys. Res., Sect. A 440, 666 (2000).

[8] A. Fomin et al., PSI Report No. TM-00-14-01, 2000 (see also http://ucn.web.psi.ch/).

[9] A. Saunders et al., Phys. Lett. B 593, 55 (2004).

[10] Y. N. Pokotilovski, Nucl. Instrum. Methods Phys. Res., Sect. A 356, 412 (1995).

[11] Y. Masuda et al., Phys. Rev. Lett. 89, 284801 (2002).

[12] C. A. Baker et al., Phys. Lett. A 308, 67 (2003).

[13] The LANSCE neutron EDM experiment, http:// p25ext.lanl.gov/edm/edm.html.

[14] R. Golub and J. M. Pendlebury, Phys. Lett. 53A, 133 (1975).

[15] E. Korobkina, R. Golub, B. W. Wehring, and A. R. Young, Phys. Lett. A 301, 462 (2002).

[16] W. Schott et al., Eur. Phys. J. A 16, 599 (2003).

[17] P. R. Huffman et al., Nature (London) 403, 62 (2000).

[18] W.-M. Yao et al. (Particle data group), J. Phys. G 33, 1 (2006).

[19] P. Ageron, W. Mampe, R. Golub, and J. M. Pendlebury, Phys. Lett. 66A, 469 (1978).

[20] A. I. Kilvington et al., Phys. Lett. A 125, 416 (1987).

[21] P. Schmidt-Wellenburg and O. Zimmer, Cryogenics 46, 799 (2006).

[22] R. Golub et al., Z. Phys. B 51, 187 (1983).

[23] R. Golub, D. Richardson, and S. K. Lamoreaux, UltraCold Neutrons (IOP Publishing Ltd., New York, 1991).

[24] V.K. Ignatovich, The Physics of Ultracold Neutrons (Clarendon Press, Oxford, 1990).

[25] K. Zeitelhack et al., Nucl. Instrum. Methods Phys. Res., Sect. A 560, 444 (2006).

[26] O. Zimmer, Nucl. Instrum. Methods Phys. Res., Sect. A 554, 363 (2005). 